\title{
An Efficient Threshold Prediction Scheme for Wavelet Based ECG Signal Noise Reduction Using Variable Step Size Firefly Algorithm
}

\author{
Vinu Sundararaj \\ ${ }^{1}$ Anna University, Chennai \\ * Corresponding author’s Email: vinovinu2020@gmail.com
}

\begin{abstract}
Electrocardiographic (ECG) signal is significant to diagnose cardiac arrhythmia among various biological signals. The accurate analysis of noisy Electrocardiographic (ECG) signal is very motivating challenge. Prior to automated analysis, the noises present in ECG signal need to be eliminated for accurate diagnosis. Many researchers have been reported different methods for denoising the ECG signal in recent years. In this paper, an optimized threshold mechanism is proposed for wavelet based medical signal noise reduction. This scheme is based on a variable step size firefly algorithm (VSSFA) in dual tree complex wavelet scheme, in which the VSSFA is utilized for threshold optimization. This approach is evaluated on several normal and abnormal ECG signals of MIT/BIH arrhythmia database, by artificially adding white Gaussian noises with variation of $5 \mathrm{~dB}$ and $10 \mathrm{~dB}$. Simulation result illustrate that the proposed system is well performance in various noise level, and obtains better visual quality compare with other methods.
\end{abstract}

Keywords: Electrocardiogram; DTCWT; VSSFA; MIT/BIH arrhythmia; Thresholding; variable step size; noise reduction.

\section{Introduction}

The computer-aided electrocardiogram (ECG) analysis has emerged as a very significant factor in the most active domains in health informatics applications [1-5]. In the past, the noise decrease in the ECG signals has riveted enhanced enthusiasm leading to the launch of the order statistics filter, low-pass filter, Kalman filter and Wiener filter, to name a few. [7-10]. Though all these devices have more or less succeeded in getting rid of the noises in varying proportions, they are found to lose signal details in the filtering process, thereby leading to the blurring, mainly on account of the fact that these filters deploy the integer-order integral to configure their relative denoising model directly or indirectly, which adversely affects the high frequency data parked in the original signal [11]. Hence, this leads to a loss of detailed information during signal denoising. In past decades, wavelet transform (WT) [12-16] has been applied to signal denoising. In addition, various wavelet based denoising methods have been developed in recent years. Poornachandra $S$ [17] elegantly launched an adaptive universal threshold based wavelet thresholding for ECG signals, which was utilized for the resurgence of the signal tainted by the white additive Gaussian noise. Alfaouri M and Daqrouq K [18] have proficiently presented a wavelet thresholding scheme employing the Daubechis $(\mathrm{dB})$ wavelet for ECG signals. Reddy GU et al. [19] deftly designed an ECG de-noising mechanism employing the enhanced thresholding based on Wavelet transform.

In the light of the above factors, an effective system for noise reduction have designed in the backdrop of diverse categories of noise, where an optimal threshold is chosen for each sub band at diverse scales by means of the Variable Step size Firefly Algorithm (VSSFA). The novel technique employs the duel tree complex wavelets which, in relation to the classical wavelets, permits a faster execution of the wavelet transforms and furnish incredible flexibility in the generation of the wavelets. After offering a comprehensive account of the technique, the proposed scheme is evaluated on ECG signal from MIT/BIH arrhythmia database for the performance evaluation. This paper is structured as following parts. In section 2, the optimization problem is provided. In Section 3, the VSSFA-based threshold selection for noise reduction scheme is 
described. The exhaustive experiments and discussion are reported in section 4. Lastly, a few concluding remarks is provided in Section 5.

\section{Problem Formulation}

Commonly, a noise reduction issue can be successfully tackled by extracting the preferred signals from noisy observations in such a manner that features of significance are conserved. In this research, a denoising model is configured as $\hat{A}=A+N$, in which $A$ and $N$ denote the original signal and noise signal correspondingly and $\hat{A}$ represents signal contaminated with noise. The denoising investigation in this paper is based on the above technique. Of late, the wavelet-based noise reduction has established itself as a wellacknowledged technique extensively employed in the evaluation of the ECG signals. The contributing factors for its zooming significance are detailed as follows: (a) the ease of execution of the technique, (b) reduced computational complication related to the approach, and (c) the skills with which the signal is assessed in diverse frequency bands linked with various signal features.

Nevertheless, the engineering experiences exhibit the fact that several categories of wavelet functions possess diverse time-frequency distributions, making it very hard to select the best wavelet function for extracting preferred features from a specified signal. Further, an improper wavelet ultimately leads to the decrease in the precision of the signal recognition. Hence, it is highly essential to design novel techniques to shape the adaptive wavelet functions to overshoot these constraints. Numerous of the wavelet based denoising algorithms use DWT (Discrete Wavelet Transform) in the decomposition stage are suffering from shift variance and lack of directionality.

The imperative need for improving the wavelet transform has led to the launch of the dual tree complex wavelet transform, which decays the image and performs the shrinkage function to eradicate the noise from the noisy image. Compared with classical wavelet transform, dual tree complex wavelet transform (DTCW) is a faster wavelet transform. The dual-tree complex wavelet transform (DTCWT) is a relatively recent enhancement to the discrete wavelet transform (DWT), with important additional properties: It is nearly shift invariant and directionally selective in two and higher dimensions. It achieves this with a redundancy factor of only 2D for $d$-dimensional signals, which is substantially lower than the Stationary DWT [20]. Extension of the DTCWT to two dimensions is achieved by separable filtering along columns and then rows. In the DTCWT, it is filtered with complex conjugates of the row (or column) filters in order to retain a second (or fourth) quadrant of the spectrum [21]. The dual tree complex DWT of a signal $x(n)$ is computed using two critically-sampled DWTs in parallel to the same data as shown in the following figure (Fig. 1). If the same filters are used in the upper tree and lower tree nothing is gained. So the filters in this structure will be designed in a specific way that the sub bands of upper DWT are interpreted as real part of complex wavelet transform and the lower tree as imaginary part as shown in figure 1 . The transform is expansive by factor 2 and shift invariant [22].

From the depiction furnished above, it is crystal clear that the DTCWT technique may be effectively employed to extort the features from a specific database and for the noise decrease in the ECG signals. According to, two significant techniques are used to shrink the wavelet coefficients like as hard and soft thresholding. In this regard, Donoho [6] made important contributions to the research of threshold selection and developed a universal threshold

$$
T h r=\sqrt{2 \log _{2}(L)}
$$

This threshold value predominantly relies on the length $L$ of the data sample and thus when $L$ is very insignificant, the outcomes continues to be noisy. If it is incredibly large, very significant signals are got rid of. Hence, the choice of the threshold values has a very great impact on the noise reduction outcome. To outsmart this issue, in this paper, a novel noise reduction technique based on a Variable Step Size Firefly Algorithm (VSSFA) is proposed with the intention of segregating from the ECG signals, whereas the VSSFA is effectively employed to achieve the optimal threshold values in the dual tree complex wavelet structure. 


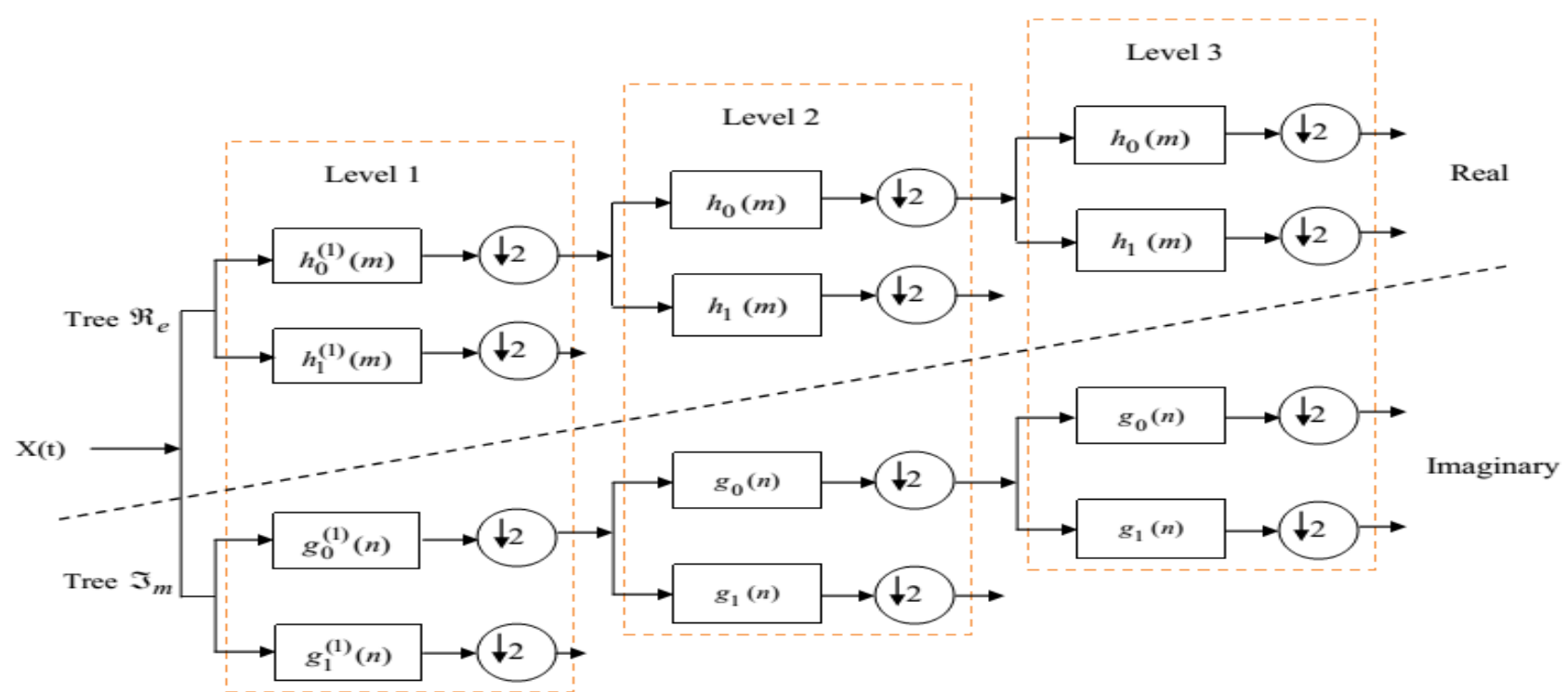

(a)

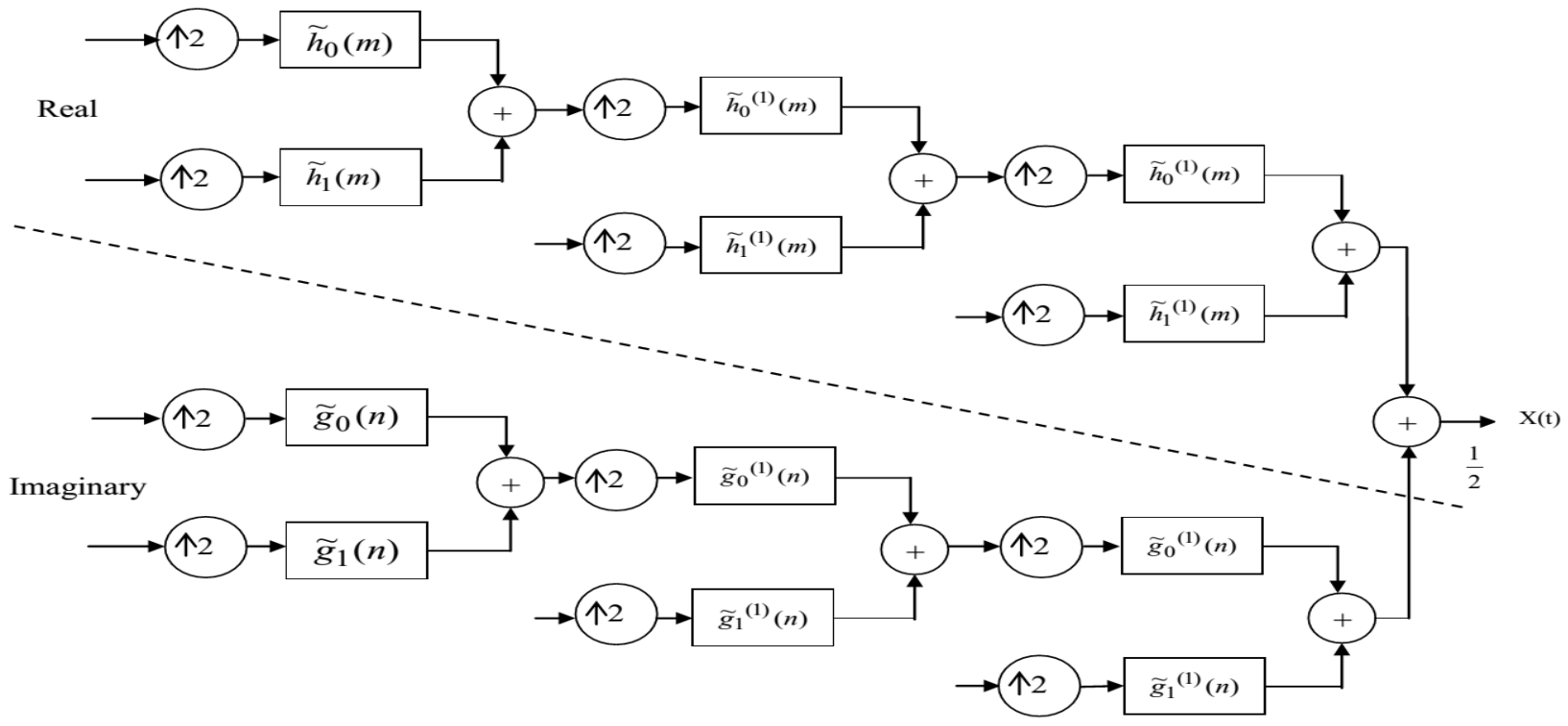

(b)

Figure.1 Three levels Dual tree complex wavelet transform (a) decomposition, (b) reconstruction

\section{Optimized noise reduction scheme via firefly algorithm}

Standard Firefly Algorithm (SFA) still has some limitations. In standard firefly algorithm (FA), the most important parameter that moves the current position towards the optimum position is the step $\sigma$. But the method of setting step $\sigma$ is static effectively, which can't be adaptive to different problems. Firstly, each firefly has the same step settings and may fall into the local optimum, resulting in premature convergence. Secondly, a large step $\sigma$ can skip the best optimal solution when it is in the vicinity of the firefly during the early part of the search, thereby reducing search performance of the best optimal solution. Finally, from Equation (5), it is useful for fireflies to explore new search area with a large step $\sigma$. However, it is not helpful to the convergence of global optimal. If step $\sigma$ with a small value, the result is opposite. Therefore, the step $\sigma$ has a great impact on the exploration and convergence of the firefly algorithm. The step $\sigma$ should decrease slowly with the large number of generations while decrease rapidly with the small generations, and there is no doubt about the effectiveness on the exploration and convergence of the algorithm. To take into account the above problems and increase the algorithm's search ability, the algorithm should be redefined in the manner that the step of the fireflies should be dynamic and changeable. 


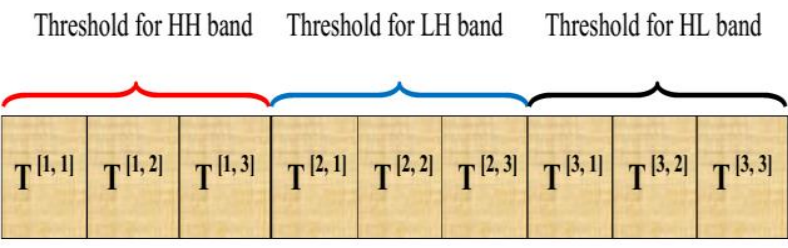

Figure.2 The solution encoding for the VSSFA-based dual tree complex wavelet transform

According to these challenges, the authors [23] have developed a variable step size firefly algorithm (VSSFA) to improve the standard firefly algorithm (SFA) performance. Motivated by this study, the VSSFA based threshold optimization framework is introduced in the dual tree complex wavelet transform for noise denoising. The VSSFA based threshold optimization in wavelet is introduced in this paper and briefly explained as follows.

\subsection{Solution or Firefly Encoding}

Since real-coding approach is adequate when tackling optimization problems in continuous domains, real-coding is used to code the threshold values which lie in continuous search space. Initially, the Electrocardiogram (ECG) signals are segmented into ten sub-bands by means of a three-scale DTCWT transform, thus four frequency sub-bands including low-low (LL), low-high (LH), high-low (HL) and high-high (HH) are obtained at each scale.

In this case, since three frequency sub-bands need to be shrunk, each firefly represents a ninedimensional real-valued vector, $T=\left\{T_{j}^{l}, T_{j}^{2} \ldots T_{j}^{9}\right\}$, in which, $j$ symbolizes the $j^{\text {th }}$ solution in the population $X$. Its each element signifies a threshold value $T(s$, $c$ ) assigned to the sub-band $s$ at the scale $c$ for every firefly, where $1 \leq s \leq 3,1 \leq c \leq 3, c$ denotes the degree of decay of the DTCWT wavelet, and $s$ corresponds to the sub-band at scale $c$. The dual tree complex wavelet threshold based firefly encoding is elegantly exhibited in figure 2, in which, $T^{[1,1]}, T^{[1,2]}, T^{[1,3]}$ characterizes the threshold values for scale 1,2 and 3 respectively.

\subsection{Fitness Computation}

For the purpose of estimating the fitness of a solution, it is necessary to design an objective function to measure the performance of each solution. In this research, the objective function is formulated as:

$$
\text { Fit }=\max (S N R-R M S E)
$$

$$
\begin{array}{r}
S N R=10 \times \log _{10}\left[\frac{\sum_{n=1}^{L}\left[\hat{A}^{[s, c]}\right]^{2}}{\left.\sum_{n=1}^{L} A^{[s, c]}-\hat{A}^{[s, c]}\right)^{2}}\right] \\
R M S E=\sqrt{\frac{1}{2 L} \sum_{n=1}^{L-1}\{\hat{A}-A\}^{2}}
\end{array}
$$

Where, $\mathrm{A}^{[s, c]}$ and $\hat{\mathrm{A}}^{[s, c]}$ are the input noisy and denoised signals, respectively at particular sub-band $s$ and scale $c$;

In addition, from equation (3) and (4),

$$
\begin{gathered}
\hat{A}^{[s, c]}=W^{-1}\left(A_{d t c w d}^{[s, c]}\right) \\
A_{d t c w d}^{[s, c]}=T_{s o f t}\left(A_{d t c w}^{[s, c]}, T h r^{[s, c]}\right) \\
A_{d t c w}^{[s, c]}=W(A)
\end{gathered}
$$

Where, $W$ and $W^{-1}$ are the dual tree complex WT and inverse dual tree complex WT operators; $A_{d t c w}{ }^{[s, c]}$ and $A_{d t c w d}{ }^{[s, c]}$ denote dual tree complex wavelet and threshold applied coefficients across sub-band $s$ and level $c$; and $T_{s o f f}\left(A_{l w}{ }^{[s, c]}, T h r^{[s, c]}\right)$ denotes the soft threshold operator of DTCWT coefficient $A_{d t c w}{ }^{[s, c]}$ with threshold $T h r^{[s,}{ }^{c]}$ at particular sub-band $s$ and scale $c$.

\subsection{Firefly Updation}

To generate new fireflies, a variable step size firefly operator is used, which decreases nonlinearly from iteration to iteration. By using this strategy to adjust the step of Firefly Algorithm (FA) can achieving a good balance between exploration and exploitation.

The movement of the firefly $i$, when attracted to another more attractive (brighter) firefly $j$, is determined by

$$
X_{i}=X_{i}+\beta_{0} e^{-\gamma r_{i, j}^{2}}\left(X_{i}-X_{j}\right)+\sigma\left(\text { rand }-\frac{1}{2}\right)
$$

The second term in equation (8) is due to attraction, the third term introduces randomization with $\sigma$ being the randomization parameter and "rand" is a random number generated uniformly distributed between 0 and 1 .

The attractiveness $\beta(r)$ can be any monotonically decreasing function such as the following form:

$$
\beta(r)=\beta_{0} e^{-\gamma r^{2}}
$$

Where, $\beta_{0}$ is the attractiveness at $r=0, r$ is the distance between two fireflies. The distance function is given as follows:

$$
r_{i, j}=\left\|X_{i}-X_{j}\right\|=\sqrt{\sum_{k=1}^{d}\left(x_{i, k}-x_{j, k}\right)^{2}}
$$

Where, $x_{i, k}$ is the $k^{\text {th }}$ component of the spatial coordinate of the $i^{\text {th }}$ firefly and $d$ is the total number 
of dimensions. Also, $i \in\left\{1,2, \ldots, F_{n}\right\}$ is randomly chosen index. Although $j$ is determined randomly, it has to be different from $i$. Here, $F_{n}$ is the number of fireflies.

\subsection{Computation of Variable Size $\sigma$}

In standard FA, the method of setting step $\sigma$ is static. It cannot really reflect the searching process. In general, it is useful for fireflies to explore new search space with a large step, but it is not helpful to the convergence of global optimum. If step has a small value, the result is contrary. Therefore, the step $\sigma$ has a great affect on the exploration and convergence of the algorithm. It would be beneficial to balance the ability of global exploration and local exploitation, and it should also be concerned with its current situation. For this reason, a dynamic adjusting scheme of step $\sigma$ is designed which can be controlled. According to, a nonlinear equation is used and designs a dynamic adjusting scheme of step, which helps to balance the ability of global exploration and local exploitation. The step $\sigma$ can be calculated as following:

$$
\sigma(t)=\frac{0.4}{\left(1+\exp \left(\frac{0.015 *(t-\text { Maxgen })}{3}\right)\right)}
$$

In equation (11), $t$ is the current number of iterations; Maxgen is the maximal number of iterations. New individuals $N_{x}$ are obtained by using above-mentioned steps and go to next iteration.

\subsection{Termination Criteria}

The maximum numbers of generation are employed as a stopping criterion and register the optimum in each generation.

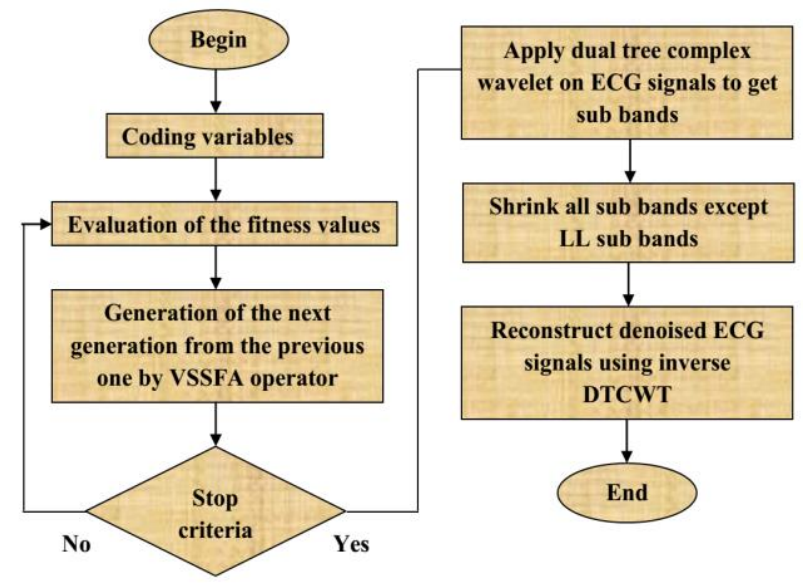

Figure. 3 The flow diagram of the VSSFA-based noise reduction method

\subsection{Algorithm Description}

The significant steps of the VSSFA-based noise reduction approach for ECG signals are summarized as follows:

Step 1: The ECG signals are taken from MIT/BIH arrhythmia data base [24]. This database contains 48 different ECG signals (tapes) with 30 minute duration which are sampled at $360 \mathrm{~Hz}$ frequency. For experimentation, additive white Gaussian noise of $5 \mathrm{~dB}$ and $10 \mathrm{~dB}$ SNR are artificially added with randomly selected 10 tapes of ECG data records where 5 records are normal and remaining are abnormal.

Step 2: Generate the initial population of Fireflies, $m$.

Step 3: Evaluation of the fitness of each firefly in the population

Step 4: Creation of the next generation from the previous one by Variable step size firefly algorithm (VSSFA).

$>$ Calculate intensity for each firefly member

$>$ Calculate the step $\sigma$ according to Equation (11);

$>$ Move each firefly towards other brighter fireflies; the position of each firefly is updated by Equation (8);

$>$ Update the solution set;

Step 5: If the execution condition is met, then stop; otherwise, go to Step 3.

Step 6: Apply dual tree complex wavelet transform (DTCWT) on ECG signals to get subbands.

Step 7: By the optimized thresholds, the shrinking process is applied on all sub-bands except LL sub-bands.

Step 8: Apply inverse dual tree complex wavelet transform (DTCWT) to reconstruct denoised ECG signals. The flowchart of the VSSFA-based denoising method is illustrated in 3.

\section{Result and Discussion}

In this section, the proposed noised reduction system is verified through the evolutionary process as well as the quantitative metrics. The experiments are run with 4GB-RAM, WIN-7 OS and MATLAB 2015a software. The performance of the proposed method is evaluated based on the five matrices SNR, RMSE, NRA, GP and BIAS.

\section{Signal-to-Noise Ratio (SNR)}




$$
S N R=10 \times \log _{10}\left[\frac{\sum_{n=1}^{L} \hat{A}(t)^{2}}{\sum_{n=1}^{L}(A(t)-\hat{A}(t))^{2}}\right]
$$

Root Mean Square Error (RMSE):

$$
R M S E=\sqrt{\frac{1}{2 L} \sum_{n=1}^{L-1}\left\{\left(\hat{A}(t)-(A(t)\}^{2}\right.\right.}
$$

\section{Noise reduction Accuracy (NRA):}

It reflects the correlation of the signal,

$$
N R A=\frac{\sum_{n=1}^{L}\left\{\left(\hat{A}(t)-h_{\text {med }}\right)\left(A(t)-A_{\text {med }}\right)\right\}}{(L-1) S_{\hat{A}} S_{A}} * 100
$$

Gain Parameter: The ratio of the original noise and the remainder noise:

$$
G P=10 \times \log _{10}\left[\frac{\operatorname{var}((A(t))}{\operatorname{var}((\hat{A}(t)-A(t))}\right]
$$

Bias: It reflect the average systematic deviation of noise reduction signal and the original signal

$$
B I A S=\frac{1}{L} \sum_{n=1}^{L}\{\hat{A}(t)-A(t)\}
$$

Where, $A(t)$ is the original ECG signal, $\hat{A}(t)$ is the reconstructed ECG signal, $A_{\text {med }}$ is the average of $A, S_{\hat{A}}$ and $S_{A}$ is the standard deviation. $L$ is the length of the signal and var () is the variance of the variable.

In the experimental purpose, the 10 tapes of ECG signal which are selected from MIT/BIH database contain 5 tapes from normal that is randomly selected from 100-124 series and remaining 5 tapes are randomly selected from 200234 series. White gaussian noise is added with each ECG signals that results $5 \mathrm{~dB}$ and $10 \mathrm{~dB}$ SNR. The graphical representation of original, noisy and denoisy ECG signal is shown in Figure 4.

Fifteen trials are done using the VSSFA based proposed scheme in dual tree complex wavelet transform, and after the completion of each trial, the SNR, RMSE, NRA, and BIAS values of the denoised ECG signal (Tape no.201) are evaluated as quantitative metrics. Table 1 lists the computation outcome of the quantitative metrics after the trials on the ECG signals with the VSSFA in dual tree complex wavelet transforms. From table 1, it can see that after using the proposed approach, the mean of the SNR is 17.0295, which indicates that an improvement of 6.4145 is obtained and the SNR reaches the maximum value of 18.715 at the seventh trial. And it also determines that the mean of the RMSE is 0.0963 and the minimum RMSE is obtained as 0.0015 at the eleventh trail. The best improvement of the RMSE is 0.5010. Meanwhile, NRA, GP and BIAS also achieved better outcome. The lowest deviation of the BIAS is achieved four times as 0.0001 . The best improvement of the NRA value is 99.63 in fourteen trials. The best value of GP parameter is achieved two times as 10.096 in fifth and fifteen trials.

Table 1. The quantitative method of metrics after the trials on ECG signal with additive white Gaussian noise

of $10 \mathrm{~dB}$ SNR (Tape no.201) with the VSSFA based noise reduction method. The bold values are the highest or lowest values in the corresponding quantitative indexes in the fifteen trials

\begin{tabular}{|cccccc|}
\hline $\begin{array}{c}\text { Number } \\
\text { of trials }\end{array}$ & SNR & RMSE & NRA & GP & BIAS \\
\hline None & 10.615 & 0.5025 & 60.21 & 6.584 & 0.0034 \\
\hline Run 1 & 17.125 & 0.2455 & 99.14 & 10.086 & 0.0008 \\
\hline Run 2 & 17.918 & 0.2405 & 98.11 & 10.095 & 0.0005 \\
\hline Run 3 & 17.515 & 0.1415 & 99.14 & 10.094 & 0.0006 \\
\hline Run 4 & 16.925 & 0.1115 & 99.24 & 10.086 & 0.0006 \\
\hline Run 5 & 17.415 & 0.0405 & 99.13 & 10.090 & $\mathbf{0 . 0 0 0 1}$ \\
\hline Run 6 & 17.515 & 0.0915 & 99.24 & 10.095 & 0.0004 \\
\hline Run 7 & $\mathbf{1 8 . 7 1 5}$ & 0.1425 & 99.47 & 10.086 & 0.0006 \\
\hline Run 8 & 16.715 & 0.0525 & 99.50 & $\mathbf{1 0 . 0 9 6}$ & $\mathbf{0 . 0 0 0 1}$ \\
\hline Run 9 & 17.915 & 0.0515 & 99.75 & 10.095 & 0.0005 \\
\hline Run 10 & 16.815 & 0.0415 & 99.04 & 10.094 & 0.0006 \\
\hline Run 11 & 17.915 & $\mathbf{0 . 0 0 1 5}$ & 98.24 & 10.095 & 0.0004 \\
\hline Run 12 & 17.825 & 0.0415 & 99.65 & 10.091 & $\mathbf{0 . 0 0 0 1}$ \\
\hline Run 13 & 16.315 & 0.0525 & 98.15 & 10.094 & 0.0005 \\
\hline Run 14 & 17.315 & 0.0415 & $\mathbf{9 9 . 6 3}$ & 10.094 & 0.0005 \\
\hline Run 15 & 17.914 & 0.0425 & 99.65 & $\mathbf{1 0 . 0 9 6}$ & $\mathbf{0 . 0 0 0 1}$ \\
\hline Mean & 17.0295 & 0.0963 & 96.70 & 9.8723 & 0.0006 \\
\hline
\end{tabular}

Table 2. Parameter description of the VSSFA algorithm

\begin{tabular}{|c|c|}
\hline $\begin{array}{c}\text { Parameter } \\
\text { name }\end{array}$ & $\begin{array}{c}\text { Description of } \\
\text { parameter }\end{array}$ \\
\hline$X$ & Population \\
\hline$L$ & Signal length \\
\hline$\sigma$ & Step size \\
\hline $\boldsymbol{\theta}$ & distance \\
\hline$\gamma_{0}$ & $\begin{array}{c}\text { Absorption } \\
\text { coefficient }\end{array}$ \\
\hline Maxgen & $\begin{array}{c}\text { Initial } \\
\text { attractiveness }\end{array}$ \\
\hline & $\begin{array}{c}\text { Maximum } \\
\text { generation }\end{array}$ \\
\hline
\end{tabular}

To evaluate the proposed noise reduction scheme, VSSFA is compared with standard firefly algorithm (FA). Figure 5 compares the evolutionary processes of affected by the VSSFA versus the FA looking at the produced fitness values over the 
course of generations. The fitness values of the optimal solutions (averaged over 15 runs) are plotted for each FA by using a population dimension of 20 and a generation number of 20. From Figure 5, it is clear that the VSSFA has achieved better fitness values than the FA. In the context of noise reduction, the higher fitness value means the better the performance of noise reduction. From the figure 5, VSSFA obtain better fitness performance than FA by adjusting the parameter $\sigma$ using equation (8). The main reason is that VSSFA adopts the variable step $\sigma$ during the course of evolution. This strategy can help VSSFA to balance the ability of global exploration and local exploitation and prevent it from stagnating and prematurely converging to a local optimum. For that reason, the VSSFA can be applied to successfully decrease the noise of ECG signals in this case. The parameter description of VSSFA algorithm is tabulated in table 2.

On other hand, VSSFA based proposed method is compared with some other methods such as S- median threshold [17] and soft threshold method in terms of SNR, RMSE, NRA, GP and BIAS. The results are illustrated in Table 3 , which make it evident that when the SNR, NRA and GP values are improved, the RMSE and BIAS are reduced in respect of all the three methods. Moreover, it is illustrated that our technique generates the highest values in terms of the SNR, NRA and GP among the three techniques and the lowest values with respect to the RMSE and BIAS. According to Table 3, the curves of the SNR and RMSE can be produced with diverse noise levels $(5,10 \mathrm{~dB})$. For the noise levels of $5 \%, 10 \%, 20 \%, 30 \%, 50 \%, 70 \%, 90 \%$ and $100 \%$, respectively, the curves of the SNR and RMSE are illustrated in figure 6. From table 3 and figure 6 , the VSSFA based algorithm achieves the SNR, GP and NRA of signals and reduces the RMSE and BIAS. Overall, our VSSFA based noise reduction method is well suited for wavelet based signal noise reduction in medical field.

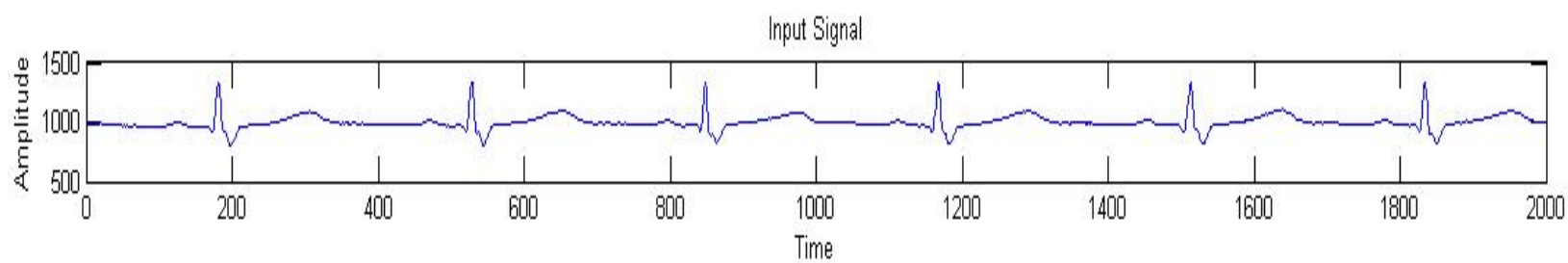

Noise Signal

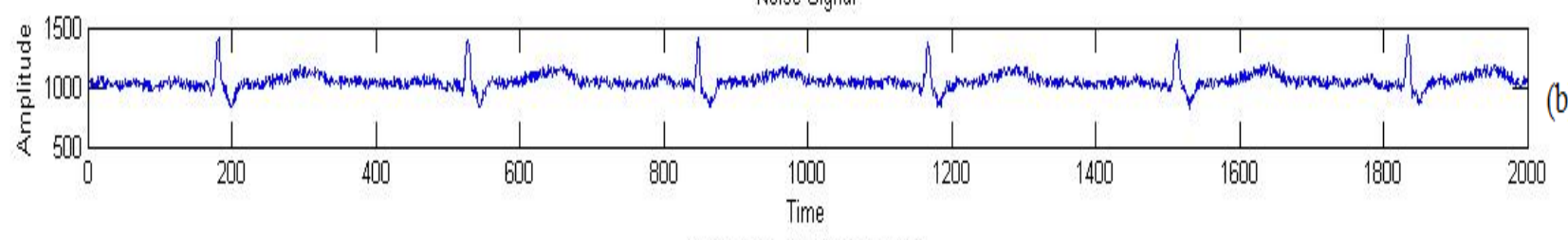

S-Median- Denoised Signal

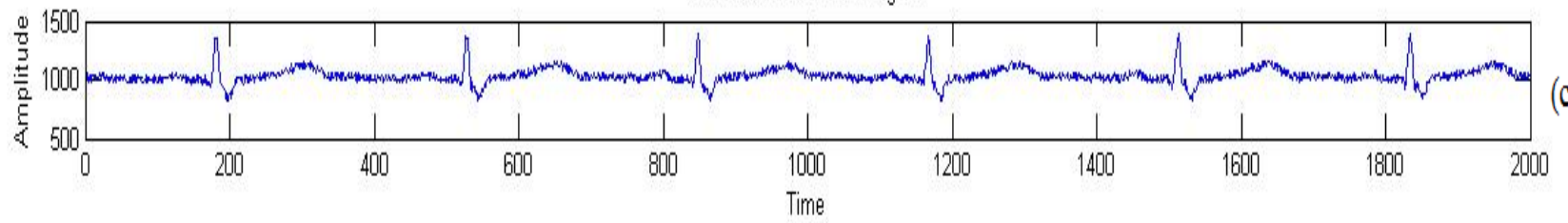

Sot Thresholding - Denoised Signal

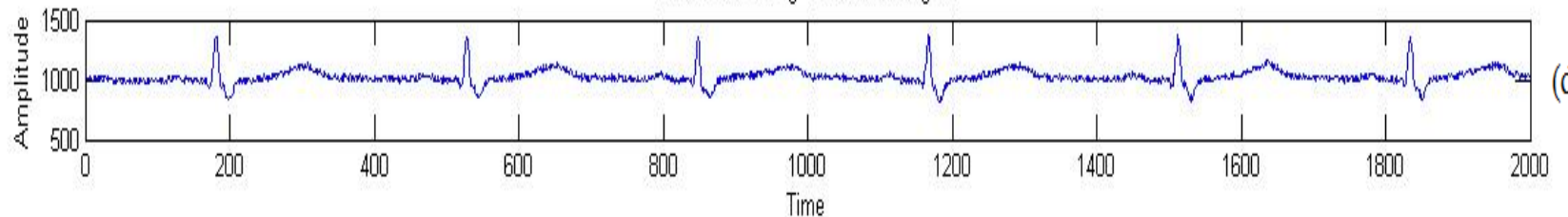

VSSFA - Denoised Signal

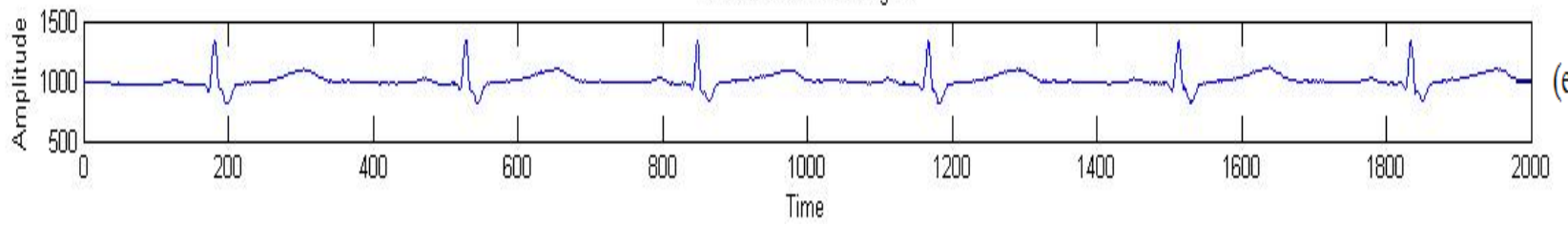

Figure.4 Visual results for (a) original ECG signal (beat No. 231), (b) noisy, (c) output signal of S-median based wavelet method [17], (d) output signal of soft threshold based method and (e) output signal of proposed method 
Table 3. The results of three denoising methods on ECG signals with addition of $5 \mathrm{~dB}$ and $10 \mathrm{~dB}$ white Gaussian noise

\begin{tabular}{|c|c|c|c|c|c|c|c|c|c|c|c|}
\hline \multirow{2}{*}{$\begin{array}{c}\text { ECG } \\
\text { Signals }\end{array}$} & \multirow{2}{*}{ Methods } & \multicolumn{5}{|c|}{$5 \mathrm{~dB}$} & \multicolumn{5}{|c|}{$10 \mathrm{~dB}$} \\
\hline & & SNR & RMSE & NRA & GP & BIAS & SNR & RMSE & NRA & GP & BIAS \\
\hline \multirow{3}{*}{231} & Soft threshold & 10.615 & 0.2025 & 53.21 & 8.584 & 0.0034 & 13.615 & 0.2025 & 60.21 & 6.588 & 0.0034 \\
\hline & $\begin{array}{c}\text { S-median } \\
\text { threshold [17] }\end{array}$ & 11.125 & 0.0655 & 96.14 & 9.086 & 0.0008 & 14.165 & 0.0434 & 96.15 & 7.082 & 0.0008 \\
\hline & VSSFA & 12.318 & 0.0015 & 98.14 & 10.096 & 0.0005 & 14.918 & 0.0242 & 97.12 & 8.093 & 0.0005 \\
\hline \multirow{3}{*}{217} & Soft threshold & 7.515 & 0.2415 & 97.14 & 8.097 & 0.0006 & 13.515 & 0.4415 & 87.24 & 7.091 & 0.0006 \\
\hline & $\begin{array}{c}\text { S-median } \\
\text { threshold [17] }\end{array}$ & 8.125 & 0.1015 & 94.24 & 8.986 & 0.0006 & 14.275 & 0.0804 & 91.24 & 8.083 & 0.0006 \\
\hline & VSSFA & 10.185 & 0.0975 & 97.13 & 10.096 & 0.0001 & 17.015 & 0.0400 & 94.13 & 9.094 & 0.0001 \\
\hline \multirow{3}{*}{214} & Soft threshold & 7.615 & 0.8025 & 90.21 & 8.584 & 0.0034 & 11.615 & 0.2025 & 89.21 & 6.584 & 0.0034 \\
\hline & $\begin{array}{c}\text { S-median } \\
\text { threshold [17] }\end{array}$ & 7.985 & 0.0765 & 96.14 & 9.086 & 0.0008 & 12.200 & 0.0589 & 95.14 & 7.085 & 0.0008 \\
\hline & VSSFA & 9.918 & 0.0405 & 98.14 & 9.096 & 0.0005 & 16.518 & 0.0095 & 96.14 & 8.199 & 0.0005 \\
\hline \multirow{3}{*}{207} & Soft threshold & 7.515 & 0.4415 & 91.14 & 8.097 & 0.0006 & 11.515 & 0.4415 & 87.14 & 7.097 & 0.0006 \\
\hline & $\begin{array}{c}\text { S-median } \\
\text { threshold [17] }\end{array}$ & 8.315 & 0.1845 & 94.24 & 9.006 & 0.0006 & 12.650 & 0.0565 & 94.34 & 9.081 & 0.0006 \\
\hline & VSSFA & 9.415 & 0.0257 & 97.13 & 10.096 & 0.0001 & 19.015 & 0.0205 & 96.13 & 10.006 & 0.0001 \\
\hline \multirow{3}{*}{201} & Soft threshold & 9.615 & 0.3025 & 90.21 & 8.584 & 0.0034 & 10.615 & 0.8025 & 90.21 & 6.584 & 0.0034 \\
\hline & $\begin{array}{c}\text { S-median } \\
\text { threshold [17] }\end{array}$ & 10.485 & 0.0655 & 96.14 & 8.986 & 0.0008 & 13.225 & 0.0480 & 96.24 & 7.080 & 0.0008 \\
\hline & VSSFA & 12.918 & 0.0305 & 91.14 & 9.096 & 0.0005 & 15.098 & 0.0215 & 97.14 & 8.093 & 0.0005 \\
\hline \multirow{3}{*}{113} & Soft threshold & 6.515 & 0.4415 & 89.14 & 7.097 & 0.0006 & 10.515 & 0.4415 & 87.12 & 8.097 & 0.0006 \\
\hline & $\begin{array}{c}\text { S-median } \\
\text { threshold [17] }\end{array}$ & 7.115 & 0.1315 & 94.24 & 8.086 & 0.0006 & 11.450 & 0.0995 & 91.22 & 9.085 & 0.0006 \\
\hline & VSSFA & 9.515 & 0.0905 & 96.13 & 9.096 & 0.0001 & 13.680 & 0.0405 & 94.13 & 10.095 & 0.0001 \\
\hline \multirow{3}{*}{103} & Soft threshold & 7.615 & 0.3025 & 90.21 & 6.584 & 0.0034 & 10.615 & 0.3025 & 90.21 & 8.587 & 0.0034 \\
\hline & $\begin{array}{c}\text { S-median } \\
\text { threshold [17] }\end{array}$ & 8.345 & 0.1245 & 96.14 & 7.086 & 0.0008 & 11.716 & 0.0816 & 96.11 & 9.082 & 0.0008 \\
\hline & VSSFA & 9.828 & 0.0705 & 98.14 & 8.096 & 0.0005 & 14.518 & 0.0405 & 97.14 & 10.091 & 0.0005 \\
\hline \multirow{3}{*}{102} & Soft threshold & .915 & 0.2415 & 87.14 & 6.097 & 0.0006 & 9.515 & 0.4415 & 87.12 & 8.067 & 0.0006 \\
\hline & $\begin{array}{c}\text { S-median } \\
\text { threshold [17] }\end{array}$ & 6.905 & 0.0725 & 94.24 & 7.086 & 0.0006 & 10.895 & 0.0530 & 93.24 & 9.087 & 0.0006 \\
\hline & VSSFA & 8.495 & \begin{tabular}{|l|}
0.0475 \\
\end{tabular} & 98.13 & 8.096 & 0.0001 & 12.355 & 0.0301 & 94.13 & 10.096 & 0.0001 \\
\hline \multirow{3}{*}{101} & Soft th & 6.615 & 0.2025 & 89.21 & 6.584 & 0.0034 & 10.615 & 0.2025 & 90.31 & 8.584 & 0.0034 \\
\hline & $\begin{array}{c}\text { S-median } \\
\text { threshold [17] }\end{array}$ & 7.095 & 0.0865 & 96.14 & 8.086 & 0.0008 & 11.405 & 0.0617 & 96.14 & 9.087 & 0.0008 \\
\hline & VSSFA & 7.918 & 0.0505 & 98.14 & 9.096 & 0.0005 & 12.938 & 0.0301 & 97.19 & 10.096 & 0.0005 \\
\hline \multirow{3}{*}{100} & Soft threshold & 7.005 & 0.2415 & 87.14 & 7.097 & 0.0006 & 9.515 & 0.4415 & 87.14 & 9.076 & 0.0006 \\
\hline & $\begin{array}{c}\text { S-median } \\
\text { threshold [17] }\end{array}$ & 7.615 & 0.0815 & 94.24 & 7.589 & 0.0006 & 10.805 & 0.0575 & 92.87 & 10.083 & 0.0006 \\
\hline & VSSFA & 8.105 & 0.0535 & 98.13 & 8.096 & 0.0001 & 12.515 & 0.0295 & 94.13 & 10.096 & 0.0001 \\
\hline
\end{tabular}




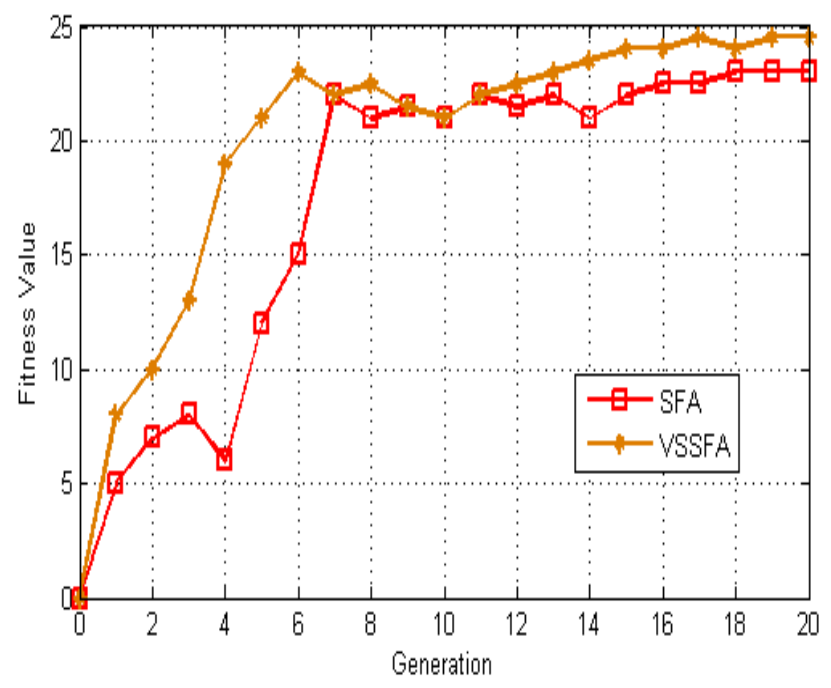

Figure.5 The evolution curve of the fitness value on ECG signal (Tape number 231) with VSSFA and SFA

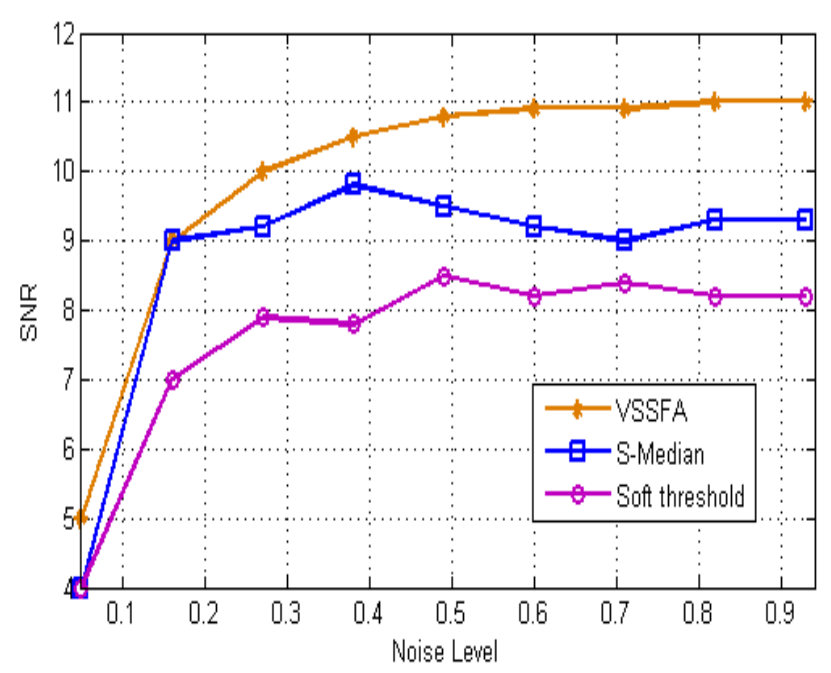

(a)

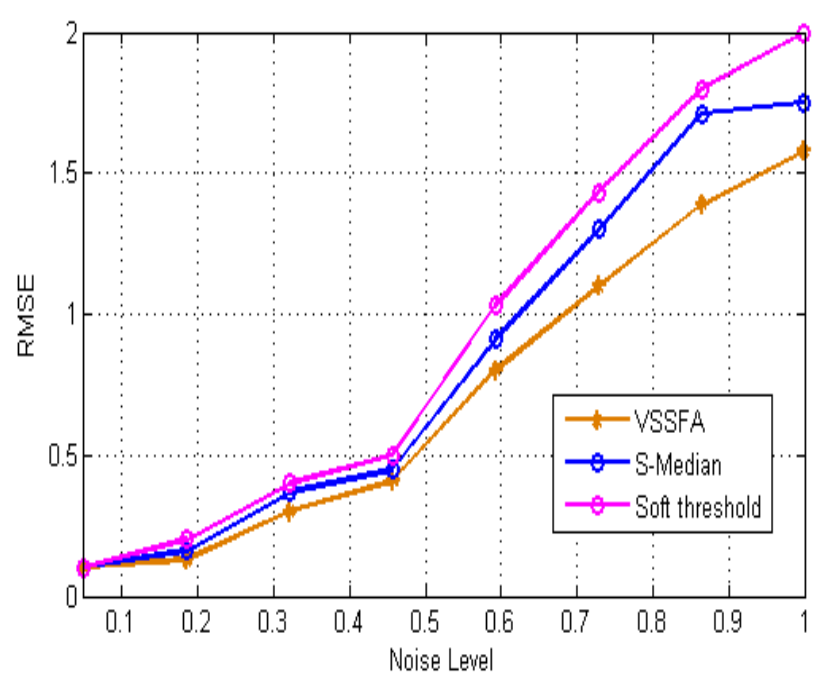

(b)

Figure.6 The SNR and RMSE curves with various noise levels. (a) The SNR curve. (b) The RMSE curve
To further test the performance of the proposed scheme, the QRS detection algorithm is applied on the denoised ECG signal. The performances of the different methods are computed by the sensitivity $(\mathrm{Se})$ and the positive predictivity $(+P)[25,26]$. These parameters are defined by

$$
\begin{gathered}
S e=\frac{T P}{T P+F N} \\
+P=\frac{T P}{T P+F P}
\end{gathered}
$$

Table 4. QRS detection sensitivity and positive productivity of the original and denoised ECG signals

\begin{tabular}{|c|c|c|c|c|c|c|}
\hline \multirow{2}{*}{ Tape No } & \multicolumn{2}{|c|}{ Original } & \multicolumn{2}{|c|}{ S-Median $[17]$} & \multicolumn{2}{c|}{ Proposed } \\
\cline { 2 - 7 } & $S e(\%)$ & $+P$ & $S e(\%)$ & $+P$ & $S e(\%)$ & $+P$ \\
\hline 231 & 99.73 & 99.82 & 99.62 & 99.62 & 99.74 & 99.70 \\
\hline 103 & 100 & 100 & 99.62 & 99.37 & 99.72 & 99.88 \\
\hline Average & 99.865 & 99.91 & 99.62 & 99.495 & 99.73 & 99.79 \\
\hline
\end{tabular}

QRS detection is applied on denoised ECG signal using Pan-Tompkins method [27] which is shown in Table 6 . To verify the performance of the proposed method, the sensitivity and positive predictivity are computed on ECG data 103 and 231 which are randomly selected from MIT-BIH data base. The average sensitivity and positive predictivity of WT based S-median method is $99.62 \%$ and $99.495 \%$ whereas, the average sensitivity and positive predictivity of the proposed method is $99.73 \%$ and $99.79 \%$. It is seen from the Table 4 that proposed method achieved better QRS detection sensitivity and positive predictivity than other method.

\section{Conclusion}

In this paper, a ECG signal noise reduction method was proposed based on a variable step size firefly algorithm (VSSFA) for ECG signals, where the VSSFA is used to obtain the optimal threshold in dual tree complex wavelet framework. The proposed method was applied on $5 \mathrm{~dB}$ and $10 \mathrm{~dB}$ SNR where White Gaussian noise was artificially added with original ECG signal. The 10 tapes of ECG signal which are picked from MIT/BIH database include 5 tapes from normal that is randomly selected from 100-124 series and remaining 5 tapes are randomly selected from 200-234 series. QRS detection accuracy of the denoised ECG signal was compared with S-median based wavelet based method. The performance of the proposed method was evaluated through SNR, RMSE, NRA, GP and BIAS. The experimental results show that the proposed method was able to reduce noise from the noisy ECG signals more accurately and consistently in comparison to 
some of the existing methods. From the analysis, it expects that the analysis carried out in this paper can be useful for paramedics to accurately diagnose cardiovascular ailments in patients. In future, this research can be applied other wavelet families. Additionally, adaptive algorithm will be developed where the parameters are controlled adaptively to improve the parameter setting.

\section{Reference}

[1] D. J. Jagannath and A. I. Selvakumar, "Issues and research on foetal electrocardiogram signal elicitation", Biomed. Signal Process. Control, Vol. 10, pp. 224-244, 2014.

[2] E. B. Mazomenos, D. Biswas, A. Acharyya, C. Taihai, K. Maharatna, J. Rosengarten, J. Morgan, and N. Curzen, "A low-complexity ECG feature extraction algorithm for mobile healthcare applications", IEEE J. Biomed. Health Inform, Vol. 17, No. 2, pp. 459-469, 2013.

[3] C. Taihai, E. B. Mazomenos, K. Maharatna, S. Dasmahapatra, and M. Niranjan, "Design of a lowpower on-body ECG classifier for remote cardiovascular monitoring systems", IEEE J. Emerg. Select. Top. Circuits Syst, Vol. 3, No. 1, pp. 75-85, 2013.

[4] G. R. Tsouri and M. H. Ostertag, "Patient-specific 12lead ECG reconstruction from sparse electrodes using independent component analysis", IEEE J. Biomed. Health Inform, Vol. 18, No. 2, pp. 476-482, 2014.

[5] S. M. Dima, C. Panagiotou, E.B. Mazomenos, J. A. Rosengarten, K. Maharatna, J. V. Gialelis, N. Curzen, and J. Morgan, "On the detection of myocadial scar based on ECG/VCG analysis", IEEE Trans. Biomed. Eng. Vol. 60, No. 12, pp. 3399-3409, 2013.

[6] D.L. Donoho, I.M. Johnstone, De-noising by softthresholding, IEEE Transaction on Information Theory 41 (1995) 613-627.

[7] M. Dennis and J. Healy, "Modern Signal Processing", Cambridge University Press, London, 2004.

[8] L. Smital, M. Vítek, J. Kozumplík and I. Provazník, "Adaptive wavelet wiener filtering of ECG signals", IEEE Trans. Biomed. Eng, Vol. 60, No. 2, pp. 437445, 2013.

[9] O. Sayadi and M. B. Shamsollahi, "ECG denoising and compression using a modified extended Kalman filter structure", IEEE Trans. Biomed. Eng Vol. 55, No. 9, pp. 2240-2248, 2008.

[10] R. Vullings, B. D. Vries and J. W. M. Bergmans, "An adaptive Kalman filter for ECG signal enhancement", IEEE Trans. Biomed. Eng, Vol. 58, No. 4, pp. 1094-1103, 2011.

[11] G. Huang, L. Xu, Q. L. Chen and M. R. Wang, "Image denoising using fractional integral", in: IEEE International Conference on Computer Science and Automation Engineering, pp. 107-112, 2012.

[12] S. Mallat and W. L. Hwang, "Singularity detection and processing with wavelets", IEEE Transactions on Information Theory, Vol. 32, pp. 617-643, 1992.

International Journal of Intelligent Engineering and Systems, Vol.9, No.3, 2016 Available:
[13] S. Mallat and S. Zhong, "Characterization of signals from multiscale edges", IEEE Transactions on Pattern Analysis and Machine Intelligence, Vol. 14, pp. 710-732, 1992.

[14] H. Nobuhara, D. B. K. Trieu, T. Maruyama and B. Bede, "Max-plus algebra-based wavelet transforms and their FPGA implementation for image coding", Information Sciences, Vol. 180, No. 17, pp. 3232 $3247,2010$.

[15] M. Vetterli and C. Herley, "Wavelet and filter banks: theory and design", IEEE Transactions on Signal Processing, Vol. 40, pp. 2207-2232, 1992.

[16] Q. Wu and R. Law, "Complex system fault diagnosis based on a fuzzy robust wavelet support vector classifier and an adaptive Gaussian particle swarm optimization", Information Sciences, Vol. 180, No. 23, pp. 4514-4528, 2010.

[17] S. Poornachandra "Wavelet-based denoising using sub band dependent threshold for ECG signals", Digital Signal Process, Vol. 18, No. 1, pp. 49-55, 2008.

[18] M. Alfaouri and K. Daqrouq, "ECG signal denoising by wavelet transform thresholding", Am J Appl Sci, Vol. 5, No. 3, pp. 276-81, 2008.

[19] G. U. Reddy, M. Muralidhar, S. Varadarajan, "ECG de-noising using improved thresholding based on Wavelet transform", Int J Comput Sci Network Security, Vol. 9, No. 9, pp. 221, 2009.

[20] I. W. Selesnick, R. G. Baraniuk and N.C. Kingsbury, "The dual-tree complex wavelet transform", IEEE Signal Process. Mag., Vol. 22, No. 6, pp. 123-151, 2005.

[21] N. G. Kingsbury, "Image processing with complex wavelets", Philos. Trans. R. Soc. London A, Math. Phys. Sci, Vol. 357, No.1760, pp. 2543-2560, 1999.

[22] V. N. P. Raj, T. Venkateswarlu, "Denoising of medical images using dual tree complex wavelet transform", Proc. Technol, Vol. 4, pp. 238-244, 2012.

[23] S. Yu, S. Zhu, Y. Ma and D. Mao, "A variable step size firefly algorithm for numerical optimization", Applied Mathematics and Computation, Vol. 263, pp. 214-220, 2015.

[24] The MIT-BIH arrhythmias database [Online]. http://physionet.org/physiobank/database/mitdb/

[25] J. Oster, O. Pietquin, M. Kraemer and J. Felblinger, "Nonlinear bayesian filtering for denoising of electrocardiograms acquired in a magnetic resonance environment", IEEE Trans Biomed Eng 2010; Vol 57, No. 7, pp. 1628-38, 2010.

[26] J. P. Martinez, R. Almeida, S. Olmos , A. P Rocha and P. Laguna, "A wavelet-based ECG delineator: evaluation on standard databases", IEEE Trans Biomed Eng, Vol. 51, No. 4, pp. 570-81, 2004.

[27] P. J. Tompkins and J. Willis, "A real-time QRS detection algorithm”, IEEE Trans Biomed Eng, Vol. 3, pp. 230-236, 1985. 